\title{
QUANTUM MECHANICAL THEORIES OF CONSCIOUSNESS
}

Henry P. Stapp

Theoretical Physics Group

Lawrence Berkeley National Laboratory

University of California

Berkeley, CA 94720

This work was supported by the Director, Office of Science, Office of High Energy and Nuclear Physics, of the U.S. Department of Energy under contract DE-AC02-05CH11231

\section{Abstract}

Quantum mechanical theories of consciousness are contrasted to classical ones. A key difference is that the quantum laws are fundamentally psychophysical and provide an explanation of the causal effect of conscious effort on neural processes, while the laws of classical physics, being purely physical, cannot. The quantum approach provides causal explanations, deduced from the laws of physics, of correlations found in psychology and in neuropsychology..

This work was supported by the Director, Office of Science, Office of High Energy and Nuclear Physics, of the U.S. Department of Energy under contract DE-AC02-05CH11231

\section{Introduction}


Isaac Newton initiated in the seventeenth century an approach to understanding nature that, with important contributions from Clerk Maxwell and Albert Einstein, developed into what is called classical mechanics. That theory is now known to be fundamentally incorrect. It was replaced around 1926 by a profoundly different theory called quantum mechanics. A principal conceptual difference between classical mechanics and its quantum successor is that the former is exclusively physical whereas the latter is essentially psychophysical. In particular, classical mechanics is theory of a material physical world conceived to be completely specified by numbers assigned to points in space and time, and to be, moreover, dynamically complete, in the sense that the behavior of these numbers for all times is completely specified by laws and initial conditions that involve only these numbers themselves. Contrastingly, orthodox quantum mechanics brings into the dynamics certain conscious choices that are not determined by the currently known laws of physics but have important causal effects in the physical world.

The entry of these causally efficacious conscious choices into contemporary physics has led some quantum physicists to believe that an adequate scientific theory of the conscious brain must be quantum mechanical. This view is challenged by some non-physicists, who argue that quantum theory deals with microscopic atomic-level processes whereas consciousness is associated with macroscopic neuronal processes, and that the concepts of classical physics provide an adequate understanding of such macroscopic systems. 
That argument is not valid. Quantum mechanics deals with the observed behaviors of macroscopic systems whenever those behaviors depend sensitively upon the activities of atomic-level entities. Brains are such systems Their behaviors depend strongly upon the effects of, for example, the ions that flow into nerve terminals. Computations show that the quantum uncertainties in the ioninduced release of neurotransmitter molecules at the nerve terminals are large (Stapp, 1993, p.133, 152). These uncertainties propagate in principle up to the macroscopic level. Thus quantum theory must be used in principle in the treatment of the physical behavior of the brain, in spite of its size.

The entry into quantum dynamics of experiential elements, and in particular of our conscious choices, is rendered possible by the effective elimination from quantum mechanics of the classical concept of material substance. Quantum theory retains the core feature of classical physics, namely a structure of mathematical quantities assigned to points in space and time. But both the behavior and the significance of this structure is greatly altered. The mathematical structure represents no longer a classically conceived material universe but rather an informational structure that represents, in effect, the knowledge associated with psychophysical events that have already occurred, and also certain objective tendencies (propensities) for the occurrence of future psychophysical events This conceptual revision is epitomized by the famous pronouncement of Heisenberg (1958, p.100): 
"The conception of objective reality of the elementary particles has thus evaporated not into the cloud of some obscure new reality concept but into the transparent clarity of a mathematics that represents no longer the behavior of particles but rather our knowledge of this behavior."

The aim of this chapter is to explain briefly, in plain words, how this enormous change came about, how it works, and how this altered conception of the role of consciousness in physics impacts on psychology and neuroscience.

\section{Origin of quantum mechanics}

Quantum mechanics was initiated by a discovery made by Max Planck in 1900 . Planck was studying the distribution over frequencies of the radiant energy emitted from a tiny hole in a hollow container. Classical physics gave clear predictions about the dependence of this energy distribution upon the temperature of the container, but those predictions did not match the empirical facts.

Planck found that the empirical data could be accounted for if one assumed that the radiant energy associated with each given frequency was concentrated in units, or quanta, with the amount of energy in a unit being directly proportional to 
the frequency of the radiation that carried it. The constant of proportionality was measured by Planck, and is called Planck's constant.

This discovery was followed by a flood of empirical data that tested various predictions of classical physics that depended sensitively on the classical conceptions of such things as electrons and electro-magnetic radiation. The data revealed fascinating mathematical structures, which seemed to involve Planck's constant, but, like Planck's data, was essentially incompatible with the classical materialist conception of the world.

Many of the best mathematicians of the generation, men such as Hilbert, Jordan, Weyl, von Neumann, Born, Einstein, Sommerfeld, and Pauli, struggled to unravel this mystery, but it was not until 1925 that the key step was made. Heisenberg found that correct predictions could be obtained if one transformed classical mechanics into a new theory by a certain "quantization" procedure. This procedure replaced the numbers that specified the structure of the classically conceived material universe by actions. Actions differ from numbers in that the ordering of numerical factors does not matter---2 times 3 is the same as 3 times 2---whereas the order in which two actions are applied can matter.

\section{Problems of interpretation}


This replacement of numbers by actions is the mathematical foundation of quantum mechanics. But an adequate physical theory requires more than just mathematical rules. It requires also a conceptual framework that allows certain mathematical statements to be tied to human experiences. In classical mechanics the interpretive framework that ties the mathematics to experience does not disturb the mathematics. It envelops the mathematical structure but does not affect it. The basic idea of the classically conceived connection between the physically and psychologically described aspects of nature is a carry-over from the planetary dynamics that was the origin of classical mechanics: the locations of objects are regarded as being directly knowable, without producing any effects on those objects. But in quantum mechanics the numbers that in classical mechanics represent, for example, the locations of various material objects are replaced by actions. These actions are associated with the process of acquiring information or knowledge pertaining to the location of that object, and this action normally affects the state that is being probed: the act of acquiring knowledge about a system becomes entangled in a non-classical way with the information-bearing quantum mechanical state of the system that is being probed.

This elimination of the numbers that were imagined to specify the physical state of the material world, and their replacement by actions associated with the acquisition of knowledge, raises huge technical difficulties. The needed conceptual adjustments were worked out principally by Bohr, Heisenberg, Pauli, 
and Born. The center of this activity was Bohr's institute in Copenhagen, and the conceptual framework created by these physicists is called The Copenhagen Interpretation.

\section{The Copenhagen interpretation}

A key feature of the new philosophy is described by Bohr:

In our description of nature the purpose is not to disclose the real essence of phenomena but only to track down as far as possible relations between the multifold aspects of our experience. (Bohr, 1934, p.18)

...the appropriate physical interpretation of the symbolic quantum mechanical formalism amounts only to prediction of determinate or statistical character, pertaining to individual phenomena appearing under conditions defined by classical physics concepts. (Bohr, 1958, p.64).

The references to "classical physics concepts" are explained as follows:

...it is imperative to realize that in every account of physical experience one must describe both experimental conditions and observations by the 
same means of communication as the one used in classical physics. (Bohr, 1958, p.88).

The decisive point is to recognize that the description of the experimental arrangement and the recording of observations must be given in plain language suitably refined by the usual physical terminology. This is a simple logical demand since by the word "experiment" we can only mean a procedure regarding which we are able to communicate to others what we have done and what we have learnt (Bohr, 1958, p 3)

Bohr is saying that scientists do in fact use, and must use, the concepts of classical physics in communicating to their colleagues the specifications on how the experiment is to be set up, and what will constitute a certain type of outcome. He in no way claims or admits that there is an actual reality out there that conforms to the precepts of classical physics.

But how can one use jointly and consistently these two mutually inconsistent descriptions of nature? That is the problem that the Copenhagen Interpretation solves, at least for all practical purposes.

\section{Quantum dualism}


The Copenhagen solution is to divide nature into two parts. One part is the observing system, including the bodies, brains, and minds of the human beings that are setting up the experimental situations and acquiring, via experiential feedbacks, increments in knowledge. This observing part includes also the measuring devices. This observing system is described in ordinary language refined by the concepts of classical physics. Thus the agent can say "I placed the measuring device in the center of the room, and one minute later I saw the pointer swing to the right." The agent's description is a description of what he does---of what probing actions he takes---and of the experienced consequences of his actions. The descriptions in terms of the language and concepts of classical physics are regarded as part of this first kind of description.

The other part of nature is the system being probed by the classically conceived and described observing system. This probed system is described in the symbolic language of quantum mathematics.

In classical physics the classical concepts are asserted to be applicable in principle right down to the atomic level. But according to the quantum precepts the quantum mathematical description must be used for any properties of the atomic entities upon which observable features of nature sensitively depend. 
This separation between the two parts of nature is called the Heisenberg cut. Above the cut one uses experience-based classical descriptions, while below the cut one uses the quantum mathematical description.

The cut can be moved from below a measuring device to above it. This generates two parallel descriptions of this device, one classical and the other quantum mechanical. The quantum description is roughly a continuous smear of classical-type states. The postulated theoretical correspondence, roughly, is that the smeared out mathematical quantum state specifies the statistical weights of the various alternative possible classically described experienceable states. The predictions of the theory thereby become, in general, statistical predictions about possible experiences described in the conceptual framework of classical physics.

There is, however, a fly in the ointment: In order to extract statistical predictions about possible experiences, some specific probing question must be physically posed. This probing question must have a countable set of experientially distinct alternative possible responses. "Countable" means that the possible responses can be placed in one-to-one correspondence with the whole numbers $1,2,3, \ldots$, or with some finite subset of these numbers. But the number of possible classically describable possibilities is not countable: there is a continuous infinity of such possibilities. So some decision must be made as to which of the possible probing questions will be physically posed. 


\section{Conscious choices}

The mathematical structure of the theory does not specify what this question is, or even put statistical conditions on the possibilities. Thus the mathematical theory is dynamically incomplete on three counts: it fails to specify which probing question will be posed, when it will be posed, and what response will then appear. The theory does, however, assign a statistical weight (probability) to each of the alternative possible responses to any question that could be posed.

Von Neumann gave the name Process 1 to the physical posing of a probing question. He specified its general mathematical form, and sharply distinguished it from the very different Process 2 , which is the mathematically specified evolution of the quantum state in accordance with the rules specified by the quantization procedure. Process 1 events intervene abruptly, from time to time, in the orderly evolution specified by Process 2 .

How does orthodox Copenhagen quantum theory resolve this critical problem of the mathematical indeterminateness of the choices of the needed Process 1 probing actions?

\section{Quantum Agents}


This problem of the indeterminateness of the conscious choices is resolved in orthodox Copenhagen quantum mechanics by adopting a pragmatic stance. The theory is considered to be a set of rules useful to a community of communicating, conscious, observing agents imbedded in a physical universe. These agents make conscious decisions about how to probe that universe, in order to observe responses that will augment their knowledge. The difficulty mentioned above, which is that the known laws do not determine which of the possible probing questions will be physically posed, is neatly resolved by saying that this very openness allows the conscious agents to freely choose which probing questions they will physically pose. Thus the causal gap in the mathematically described structure is filled by the free choices made by conscious agents.

Bohr often emphasized the freedom of these agents to make these choices:

The freedom of experimentation, presupposed in classical physics, is of course retained and corresponds to the free choice of experimental arrangement for which the mathematical structure of the quantum mechanical formalism offers the appropriate latitude. (Bohr, 1958, p.73).

To my mind there is no other alternative than to admit in this field of experience, we are dealing with individual phenomena and that our possibilities of handling the measuring instruments allow us to make a 
choice between the different complementary types of phenomena that we want to study. (Bohr, 1958, p. 51).

These quotes highlight the key fact that selection of the Process 1 probing events is determined, within the framework of contemporary physics, not by known mathematical or physical laws but rather by free choices made by conscious agents.

\section{Von Neumann's Move}

John von Neumann formulated Copenhagen quantum mechanics in a mathematically rigorous form, and then, in order to remove ambiguities associated with the placement of the Heisenberg cut, showed that this cut could be pushed all the way up, so that the entire physically describable universe, including the bodies and brains of the agents, are described quantum mechanically. This placement of the cut does not eliminate the need for Process 1. It merely places the physical aspect of the Process 1 psychophysical event in the brain of the conscious agent, while placing the conscious choice of which probing question to pose in his stream of consciousness. That is, the conscious act of choosing the probing question is represented as a psychologically described event in the agent's mind, which is called by von Neumann (1955, p. 421) the "abstract ego". This choice is physically and functionally implemented by a Process 1 action in his brain. The psychologically described and physically 
described actions are the two aspects of a single psychophysical event, whose physically described aspect intervenes in the orderly Process 2 evolution in a mathematically well defined way.

Bohr emphasized that the laws of quantum theory should continue to be valid in biological systems, but that the latitude introduced by the severe constraints upon observation imposed by the demands of sustaining life could permit such concepts such as "teleology" and "volition" to come consistently into play. (Bohr, 1958, p.10, p.22)

\section{Interactive dualism}

Orthodox quantum theory is a theory of a type called interactive dualism, which goes back in modern philosophy to Descartes, and before that to the ancient Greeks. An interactive dualism postulates the existence of two entirely different kinds of realities, mental and physical, that interact. Mental realities have the character of feelings, broadly construed to include thoughts, ideas, perceptions, pains, joys, sorrows and all things that enter directly into our streams of conscious experiences, and are described basically in psychological language. Physical realities are elements that are described in our theories of nature in terms of mathematical qualities assigned to space-time points. 
Interactive dualism combined with the precepts of classical physics gives classical interactive dualism. This has been attacked ferociously by philosophers for over three hundred years, with an intensity that has been increasing over the past half century. Quantum interactive dualism is based, instead, on orthodox (von Neumann) quantum theory.

The first main objection to classical interactive dualism is that it postulates the existence of two entirely different kinds of things, but provides no understanding of how they interact, or even can interact. The second main objection is that the physical description is, by itself, already causally complete, giving a completely deterministic account of the evolution in time of every physically described entity, which means that the mental realities have nothing to do, and no possibility of influencing anything physical. The mental side is a "ghost in the machine" that is imagined to be pulling the levers in order to 'work its will' in the physical world, but cannot really be doing so because the behavior of the physically described universe is completely determined independently of the ghostly machinations.

Quantum interactive dualism neatly evades both objections. The answer to the first is that the form of the interaction between the mentally and physically described aspects of nature is specified in von Neumann's account of the measurement process. This account is part of a careful mathematical description of the fundamental principles of quantum theory, and of how they are to be employed in practice. The specification of the form of the interaction between 
the two differently described aspects is an essential part of von Neumann's formulation of quantum theory. It is essential because quantum theory is specifically designed to be a tool that allows physicists to make computations that connect their experiences about setting up probing experiments to their expectations about the observable responses to these probing actions. Such a theory requires an adequate theory of measurement and observation, which von Neumann provides.

As regards the second objection, a huge essential difference between the classical and quantum dualities is that in the quantum case the physically described part is not causally complete. Something else is needed to complete the dynamics. Mental realities function both to complete the causal structure and also to undergird what the theory is basically about, namely the structural relationships between the elements in our streams of conscious experiences.

In my characterization of interactive dualism I spoke of two kinds of realities, physical and mental. Mental realities are certainly real: a presently felt pain really does exist. The experiencings of theoretical ideas in the streams of consciousness of physicists are also real happenings. But the existence in nature of real entities that have all the properties ascribed by the precepts of classical physics to, say, "electrons" would be surely denied by most quantum physicists. Quantum philosophy recommends avoiding commitment to the idea that there are realities in nature that accurately conform to our theoretical ideas 
about the physical universe. In regard to the physical it is only the descriptions themselves, and the way that they are used, that are ascribed significance in orthodox quantum philosophy. Ontological commitments pertaining to the physical are not part of science. In general, the practical meanings of descriptions are defined in the end by how the descriptions are used in practice.

The fact that the form of the interaction between the psychologically and physically described aspects of quantum theory is specified is important: it severely constrains the theory. Arbitrary ad hoc proposals cannot be postulated willy nilly. For example, many proposals are ruled out by the fact the living brain is large, warm, and wet, and interacts strongly with its environment. The first, and very stringent, demand on any serious proposal is that it work in this hostile-toquantum-effects setting.

The only pertinent quantum effect known to me that survives robustly under these hostile settings is the quantum Zeno effect, so-named because of its rough similarity to the paradox that claims that the hare can never catch the turtle because, by the time the hare reaches where the turtle was, the turtle will have moved on. That claim is obviously false. But there is a vaguely similar claim about quantum mechanics that is unquestionably true (Misra, 1977). If, under appropriate conditions, one repeatedly poses the same probing question at a sufficiently rapid rate, then the sequence of responses will tend to get stuck in place. In the limit of arbitrarily rapid re-posings, the response will become frozen: 
all the responses will come out to be the same, even though very strong physical forces may be working to make them change. Thus a manipulation of the timings of the probing actions, which are under the control of the consciousness of agent, can have, even in a warm, wet brain, a very special kind of physical effect. If, by mental effort, an agent can cause a sufficient increase in probing rate, then that agent can cause a state of intention and attention to be held in place much longer than would be the case if no such effort were being made.

The crucial point, here, is that the physically-described laws of quantum mechanics do not fix the times at which the physical Process 1 probing actions occur, or what these physical probing actions will be. This lacuna is the essential reason why the conscious "free choices" on the part of human agents were brought into quantum mechanics by its founders, and were retained by John von Neumann! These conscious choices control the timings of the physical Process 1 events studied by Misra and Sudarshan, and this connection entails, in principle, the capacity of these psychologically described aspects of the streams of consciousness of agents to control, via quantum Zeno holding actions, certain physically described features of the world.

Huge survival benefits could accrue to agents that can exploit this feature of the quantum mechanics, because this intentional stabilizing of attention would hold in place also the corresponding pattern of functional brain activity. 
Such a holding effect could, of course, be postulated, ad hoc, to occur in a classical-physics-based model. But in that case the holding effect would not be a direct consequence of the same basic psychophysical laws that are used by physicists to explain atomic phenomena. In the quantum case the holding effect is probably the only robust kind of effect of mind on brain that the theory predicts, whereas any desired regularity could postulated in a theory that simply adds mind ad hoc. As regards classical-physics-based theories, the view of physicists is that classical physics is an approximation to quantum physics. All effects of conscious thought upon brain activity that follow from quantum theory, such as the quantum Zeno holding effect, are eliminated in the classical physics approximation, because in that approximation the uncertainty-principle-based latitude within which the causal effects of mind upon the physically described aspects of nature operate shrinks to zero.

\section{Comparison to psychological findings}

The dynamical effect described above of a volition-induced high rapidity of the Process 1 probing actions is exactly in line with the description of the effects of volition described by William James (1892). In the section entitled Volitional effort is effort of attention he writes:

Thus we find that we reach the heart of our inquiry into volition when we ask by what process is it that the thought of any 
given action comes to prevail stably in the mind. (p. 417)

The essential achievement of will, in short, when it is most 'voluntary,' is to attend to a difficult object and hold it fast before the mind. (p.417).

Everywhere, then, the function of effort is the same: to keep affirming and adopting the thought which, if left to itself, would slip away. $($ p.421)

James may have foreseen, on the basis of his efforts to understand the mindbrain connection, the eventual downfall of classical mechanics. He closed his book with the prophetic words

... and never forget that the natural-science assumptions with which we started are provisional and revisable things. (p.433)

A lot has happened in psychology since the time of William James, but these newer developments support James's idea of the holding-attention-in-place action of volition. Much of the recent empirical and theoretical work pertaining to attention is summarized in Harold Pashler's book The Psychology of Attention (Pashler, 1998). Pashler concluded that the evidence indicates the existence of 
two distinct kinds of mental processes, one that appears not to involve volition, and that allows several perceptual processes to proceed in parallel without significant interference, and one that does involve volition and that includes planning and memory storage. This latter process seems to involve a linear queuing effect with limited total capacity.

These properties of volition-driven processes appear to be explainable in terms of the basic laws of orthodox quantum physics, which entail the existence of Process 1 physical events whose timings are controlled by conscious choices, and which can, in principle, by means of the quantum Zeno effect, tend to hold in place a pattern of neural activity that will tend to bring into being an intended effect. But this holding effect drops out in the classical-physics approximation, in which all physically described properties become completely determined by physically described properties alone, with consciousness a causally inert, or causally superfluous, bystander. Correlations between physically and psychologically described properties can be described within a classical physics based framework, but the psychologically described aspects will remain essentially epiphenomenal by-products of brain activity.

This evidence from psychology is discussed in detail in Stapp $(1999,2001)$ and in Schwartz, Stapp, and Beauregard $(2003,2005)$

\section{Application in Neuroscience}


The most direct evidence pertaining to the effects of conscious choices upon brain processes comes from experiments in which identifiable consciously controllable cognitive processes seem to be controlling directly measured physical processes in the brain. An example is the experiment of Ochsner et.al. (2001). The subjects are trained how to cognitively re-evaluate emotional scenes by consciously creating and holding in place an alternative fictional story of what is really happening in connection with a scene they are viewing.

The trial began with a $4 \mathrm{sec}$ presentation of a negative or neutral photo, during which participants were instructed simply to view the stimulus on the screen. This interval was intended to provide time for participants to apprehend complex scenes and allow an emotional response to be generated that participants would then be asked to regulate. The word Attend (for negative or neutral photos) or Reappraise (negative photos only) then appeared beneath the photo and the participants followed this instruction for $4 \mathrm{sec} \ldots$

To verify whether the participants had, in fact, reappraised in this manner, during the post-scan rating session participants were asked to indicate for each photo whether they had reinterpreted the photo (as instructed) or had used some other type of reappraisal strategy. Compliance was high: On less than $4 \%$ of trials with highly negative photos did participants report using another type of strategy. 
Reports such as these can be taken as evidence that the streams of conscious of the participants do exist and contain elements identifiable as efforts to reappraise.

Patterns of brain activity accompanying reappraisal were assessed by using functional magnetic imaging resonance (fMRI). The $\mathrm{fMRI}$ results were that reappraisal was positively correlated with increased activity in the left lateral prefrontal cortex and the dorsal medial prefrontal cortex (regions thought to be connected to cognitive control) and decreased activity in the (emotion-related) amygdala and medial orbito-frontal cortex.

How can we understand and explain the psychophysical correlations exhibited in this experiment?

According to the quantum model, the conscious feelings cause the changes in brain activity to occur. This causation is in strict conformity to the known laws of physics, as spelled out in von Neumann's book Mathematical Foundations of Quantum Mechanics.

This causal explanation, this whole causal story, falls apart if one tries to explain this psychophysical correlation within the framework of the classical approximation. That approximation entirely eliminates the effects of our conscious choices and efforts upon the physical world, including our brains. But 
what is the rational motivation for insisting on using this approximation? The applicability of the classical approximation to this phenomenon certainly does not follow from physics considerations: calculations based on the known properties of nerve terminals indicate that quantum theory must in principle be used. Nor does it follow from the fact that classical physics works reasonable well in neuroanatomy or neurophysiology: Quantum theory explains why the classical approximation works well in those domains. Nor does it follow rationally from the massive analyses and conflicting arguments put forth by philosophers of mind. In view of the turmoil that has engulfed philosophy during the three centuries since Newton cut the bond between mind and matter, the re-bonding achieved by physicists during the first half of the twentieth century must be seen as an enormous development: a lifting of the veil. Ignoring this huge and enormously pertinent development in basic science, and proclaiming the validity of materialism on the basis of inapplicable-in-this-context nineteenth century science is not a rational judgment.

Of course, one can simply abandon the idea that ideas can actually cause anything physical, and view the feeling of effort as not a cause, but rather an effect, of a prefrontal excitation that causes the suppression of the limbic response, and that is caused entirely by other purely physical activities.

Viewed from a sufficiently narrow perspective that might seem to be a satisfactory conclusion, but it leads to the old problem: why is consciousness 
present at all, and why does it feel so causally efficacious, if it has no causal efficacy at all? Why this big hoax? Quantum theory answers: There is no a hoax! It was only the premature acceptance a basically false physical theory, fundamentally inapplicable to the brain, that ever made it seem so!

The only objections I know to applying the basic principles of physics to brain dynamics are, first, the forcefully expressed opinions of some non-physicists that the classical approximation provides an entirely adequate foundation for understanding brain dynamics, in spite of the quantum calculations that indicate the opposite; and, second, the opinions of some physicists that the hugely successful orthodox quantum theory, which is intrinsically dualistic, should, for philosophical reasons, be replaced by some theory that re-converts human consciousness into a causally inert witness to the mindless dance of atoms. Neither of these opinions has any secure scientific basis.

There are several other quantum theories of consciousness, but all of them are based on von Neumann's work. The physics considerations described above rest completely on that work. I shall describe next some proposals that go far beyond von Neumann's secure base, and introduce some very controversial ideas.

\section{The Penrose-Hameroff Theory}


Roger Penrose and Stuart Hameroff (Hameroff \& Penrose, 1996) have proposed a quantum theory of consciousness that brings together three exciting but controversial ideas. The first pertains to the still-to-be-worked-out quantum theory of gravity. The second involves the famous incompleteness theorem of Gödel. The third rests upon the fairly recently discovered microtubular structure of neurons.

Penrose proposes that the abrupt changes of the quantum state that are associated with conscious experiences are generated by the gravitational effects of particles of the brain upon the structure of space-time in the vicinity of the brain. Ordinarily one would think that the effects of gravity within the brain would be too minuscule to have any significant effect on the functioning of the brain. But Penrose and Hameroff come up with an estimate of typical times associated with the gravitational effects that are in the tenth of a second range associated with conscious experiences. This fuels the speculation that the abrupt changes in the quantum state that occur in quantum theory are caused not by the entry of thoughts into brain dynamics, but by quantum effects of gravity.

But then why should thoughts or consciousness be involved at all?

Two reasons are given. Penrose uses Gödel's incompleteness theorem to argue that mental processing cannot be wholly mechanical or algorithmic. The argument takes hundreds of pages (Penrose, 1986, 1994) and has been 
attacked by many seemingly qualified critics. (e.g., Putnam, 1994). It is fair to say that it has not passed the usual demands made upon mathematical and logical arguments. But the argument claims that both mental processing and the gravitational effects are non-algorithmic, and that the latter could therefore provide in a natural way the non-algorithmic element needed for the former

The second connection of the proposed gravitational effect with consciousness is that the estimated time associated with the gravitational effect was based on the presumption that the components of the brain critical to consciousness were functioning microtubules. Data pertaining to loss of consciousness under the influence of various anesthetic agents indicate that the proper functioning of microtubules is necessary for consciousness. But many things are necessary for consciousness, so this argument that the gravitational effect is connected consciousness via microtubules is not compelling. .

A serious objection to the Penrose-Hameroff theory has been raised by Max Tegmark (2000). The Penrose-Hameroff theory requires that the critical microtubular state be a coherent quantum state that extends over a macroscopic region in the brain. Normally one expects any macroscopic coherence of a quantum state in a warm wet brain to be destroyed almost immediately. Tegmark estimates the duration of coherence to be on the order of $10^{-13}$ seconds, which is far smaller than the one tenth of a second associated with conscious events. Hagen, Hameroff, and Tuszynski (2002) have claimed that Tegmark's 
assumptions should be amended, so that the decohence time increases to $10^{-4}$ seconds, and they suggest that the remaining factors can perhaps be made up by biological factors. In any case, the need to maintain macroscopic quantum cohererence in a warm wet brain is certainly a serious problem for the PenroseHameroff model.

It might be mentioned here that in the von Neumann model described in the preceding sections quantum decoherence is an important asset, because it allows the quantum state of the brain to be understood as essentially a smeared out statistical ensemble (i.e., collection) of essentially classically conceived states, which, however, can interact with neighboring members of the ensemble in a way that preserves the quantum Zeno effect. This quasi-classical conceptualization of the quantum state of the brain allows non-physicists to have a relatively simple understanding of the mind-brain system.

\section{The Eccles-Beck approach}

An early quantum approach to the mind-brain problem was made by John Eccles (1990) who emphasized the entry of quantum effects into brain dynamics in connection with effects at nerve terminals. However, instead of building directly on the quantum rules and the profound conceptual relationships between quantum and classical mechanics he introduced a conscious biasing of the quantum statistical rules. This actually contradicts the quantum rules, thereby 
upsetting the logical coherency of the whole scheme. In a later work with Beck (2003) he retained the quantum rules, while introducing quantum uncertainties at the nerve terminals that can play the same role that they do in the standard approach described earlier. This brings the model into accord with the standard model described above, in regard to this technical point. However, Eccles added a superstructure involving conscious "souls" that can exist apart from physical brains. That suggestion goes beyond the ideas described here.

\section{Other Theories}

Several other quantum theories of consciousness have been proposed. [Bohm, 1990; Jibu, 1995]. All are outgrowths of von Neumann's formulation. The differences in these proposals are mainly at the level of technical physics. I have focused here on the over-riding general issues of why quantum theory should be relevant to consciousness in the first place, and how the switch to quantum physics impacts upon the question---vital to neuroscience, psychology, and philosophy---of the neural effects of volitional effort.

HENRY P. STAPP

Henry Stapp is a theoretical physicist with a long-time special interest in mathematical and conceptual problems in the foundations of quantum theory. He 
worked with Wolfgang Pauli and with Werner Heisenberg and has published extensively on the subjects of axiomatic S-matrix theory, quantum non-locality, philosophy of quantum theory, and the mind-brain interaction.

\section{Further Reading}

The Emperor's New Mind, Roger Penrose

Mind, Matter, and Quantum Mechanics, Henry Stapp

The Large, the Small, and the human Mind, Roger Penrose (with

Abner Shimony, Nancy Cartwright, and Stephen Hawking)

The Mind and the Brain: Neuroplasticity and the power of mental force, Jeffrey

Schwartz and Sharon Begley

The Mindful Universe, Henry P. Stapp

\section{Acknowledgements}

I thank Max Velmans and Ed Kelly for influential comments on earlier drafts.

\section{REFERENCES}


Beck, F. and J. C. Eccles 2003: Quantum processes in the brain: a scientific basis of consciousness. In N. Osaka (ed.), Neural Basis of Consciousness, 141166. Amsterdam, Philadelphia: John Benjamins

Bohm, D. J. 1990: A new theory of the relationship of mind to matter. Philosophical Psychology, 3, 271-286.

Bohr, N. 1934. Atomic Theory and the Description of Nature. Cambridge: Cambridge University Press. (Re-issued in 1961)

Bohr, N. 1958. Atomic Physics and Human Knowledge. New York: Wiley.

Eccles, J.C. 1990: A unitary hypothesis of mind-brain interaction in the cerebral cortex. Proceedings of the Royal Society of London B240, 433-451.

Eccles, J.C. 1994: How the Self Controls its Brain. Berlin, Heidelberg, New York: Springer.

Hagen, S., S. R. Hameroff, and J. A. Tuszynski 2002: Quantum computation in brain microtules: decoherence and biological feasibility. Physical Review E65, 061901-1 - 061901-11. 
Hameroff, S. R. and R. Penrose 1996: Orchestrated reduction of quantum coherence in brain microtubules: a model for consciousness. J. Consciousness Studies 3, 36-53.

Heisenberg, W. 1958: The representation of nature in contemporary physics. Daedalus 87 (Summer), 95-108.

James, W. 1892: Psychology: the briefer course. In William James: Writings 1879-1899. New York: Library of America.

Jibu, M. \& Yasue, K. 1995: Quantum brain dynamics and consciousness. Amsterdam and Philadelphia: John Benjamins.

Misra, B., and E. C. G. Sudarshan 1977: The Zeno's paradox in quantum theory. Journal of Mathematical Physics 18, 756-763.

Ochsner, K.N., S, A. Bunge, J.J. Gross, and J. D. E. Gabrieli 2002: Rethinking feelings: An fMRI study of the cognitive regulation of emotion. J. Of Cognitive Neuroscience 14:8, 1215-1229.

Pashler, H. 1998: The Psychology of Attention. Cambridge, MA: MIT Press.

Penrose, R. 1986: The Emperor's New Mind. New York: Oxford. 
Penrose, R. 1994: Shadows of the Mind. New York: Oxford.

Penrose, R. 1997: The Large, the Small, and the Human Mind. Cambbridge: Cambridge University Press.

Putnam, H. 1994: Review of Roger Penrose, Shadows of the Mind, New York Times Book Review, November 20, p.7. Reprinted in AMS bulletin: www.ams.org/journals/bull/pre-1996data/199507/199507015.tex.html

Schwartz, J. M., and S. Begley 2002: The Mind and the Brain: Neuroplasticity and the Power of Mental Force. New York: Harper-Collins.

Schwartz, J.M., H.P. Stapp, and M. Beauregard 2003: The volitional influence of the mind on the brain, with special reference to emotional self regulation. In M. Beauregard (ed.), Consciousness, Emotional Self-Regulation and the Brain. [Advances in Consciousness Research Series, Volume 54]. Amsterdam, New York: John Benjamins.

Schwartz, J.M., H.P. Stapp, and M. Beauregard 2005: Quantum theory in neuroscience and psychology: a neurophysical model of the mind-brain interaction. Phil Trans. Royal Society (Biol. Sect) (February). On line at http://www-physics.lbl.gov/ stapp/stappfiles.html 
Stapp, H. 1999: Attention, intention, and will in quantum physics. J. Consciousness Studies, 6, 143-164.

Stapp, H. 2001: Quantum theory and the role of mind in Nature. Found. Phys. 31, 1465-1499.

Tegmark, M. (2000). Importance of quantum decoherence in brain process. Physical Review E61, 4194-4206.

Von Neumann, J. (1955). Mathematical Foundations of Quantum Mechanics. Princeton: Princeton University Press. (Translated by Robert T. Beyer from the 1932 German original, Mathematiche Grundlagen der Quantummechanik. Berlin: J. Springer) 
